\title{
Sexismo ambivalente hacia hombres: Un estudio exploratorio con adolescentes mexicanos
}

\section{Ambivalent Sexism Towards Men: An Exploratory Study with Mexican Adolescents}

Aldo Alexis Arenas-Rojas. Angelópolis-Centro Universitario del Valle de Atlixco (CUVA). México

José Luis Rojas-Solís. Postdoctoral Researcher. Universidad de La Laguna



\section{Resumen}

El sexismo ambivalente hacia las mujeres ha sido investigado con mayor frecuencia en comparación con las actitudes sexistas hostiles y benevolentes hacia los hombres. Por ello en esta investigación se exploran las actitudes ambivalentes hacia hombres en adolescentes mexicanos. Método: Estudio descriptivo, transversal y cuantitativo donde cuarenta y tres mujeres y treinta siete hombres, con edades comprendidas entre 12 y 19 años $(M=16.61$, $D T=1.20)$, contestaron el Inventario de Sexismo Ambivalente hacia Hombres. Resultados: El análisis estadístico mostró que los chicos obtuvieron mayores niveles de sexismo benevolente hacia el hombre, mientras que en el sexismo hostil no se obtuvieron diferencias significativas entre hombres y mujeres. Conclusiones: Desde una perspectiva de género inclusiva es pertinente continuar con el estudio del sexismo hacia las mujeres y los hombres con el fin de entender las dinámicas subyacentes en la discriminación hacia ambos sexos. Por lo tanto, las actitudes hacia los hombres no deben ser consideradas como un tema de investigación irrelevante.

Palabras clave: Sexismo | Adolescencia

\begin{abstract}
The ambivalent sexism towards women has been investigated more frequently in comparison of the hostile and benevolent attitudes toward men. Thus, this research explores the ambivalent attitudes toward men in Mexican adolescents. Method: It is a descriptive, transversal and quantitative study. Forty-three women and thirty-seven men, aged between 12 and 19 years $(M=16.61, S D=1.20)$, answered Inventory Ambivalent Sexism towards men. Results: Statistical analysis showed that boys had higher levels of benevolent sexism towards men; while in hostile sexism no significant differences between men and women were obtained. Conclusions: From a gender inclusive perspective is pertinent to continue with the study of sexism towards women and men in order to understand the underlying dynamics discrimination towards both sexes. Thus, the attitudes toward men should not be considered as irrelevant research issue.
\end{abstract}

Keywords: Sexism | Adolescence

\section{Introducción}

El sexismo es un problema que tiene gran importancia en el ámbito social, sobre todo porque en algunos casos puede pasar desapercibido manteniendo condiciones de desigualdad y discriminación entre sexos. De allí la importancia de su detección, pues 
se le ha considerado, incluso, como un factor de riesgo para la violencia hacia la mujer (Díaz-Aguado, 2003).

El sexismo se define como "una actitud dirigida a las personas en virtud de su pertenencia a un determinado sexo biológico, función por la cual se asumen diferentes características y conductas" (Lameiras, 2002, p. 92). Mientras que el sexismo ambivalente se define como "la unión de dos creencias sexistas relacionadas: sexismo hostil y sexismo benevolente y se articula en tres componentes comunes: paternalismo, diferenciación de género y la heterosexualidad" (Glick \& Fiske, 1996, p. 494). El paternalismo se puede explicar cómo la forma en la que un padre se comporta con sus hijos, a su vez que la diferenciación de género es una justificación sobre el poder masculino ejercido en las mujeres y finalmente sitúan a la heterosexualidad como el origen de la ambivalencia en las conductas sexistas (Glick \& Fiske, 1996).

Aunque gran parte de los antecedentes disponibles sobre el sexismo ambivalente tiene por objetivo evaluar las actitudes hacia las mujeres, es importante mencionar que este tipo sexismo también podría verse reflejado hacia los hombres (Glick \& Fiske, 1999). Así, podría esperarse que algunas mujeres apoyen y justifiquen la dominación masculina y les lleve a mostrar actitudes benevolentes hacia los hombres. Otra razón, y quizá la más poderosa, es que los hombres recompensan a las mujeres que adoptan roles tradicionales y castigan a aquellas que no los cumplen, por esta razón las mujeres asumen actitudes benevolentes hacia los hombres. Además que en la mayoría de los casos dependen económicamente de los hombres, y es esta misma dependencia la que les proporcionaría razones para sentir resentimiento y respetar el poder de los hombres (Rodríguez, Lameiras, Carrera \& Faílde, 2009b).

Para la identificación de actitudes sexistas Glick y Fiske (1996) desarrollaron la Escala de Sexismo Ambivalente (ASI) diseñada para medir las actitudes ambivalentes de los hombres hacia las mujeres. De la misma manera Glick y Fiske (1999) desarrollaron la Escala de Ambivalencia hacia los hombres (AMI) creada para medir los prejuicios hostiles y benevolentes que las mujeres tienen hacia los hombres. Esta escala está formada por 20 ítems que evalúan la hostilidad y benevolencia hacia los hombres. El sexismo hostil está compuesto por tres componentes: resentimiento paternalista, diferenciación de género compensatoria y hostilidad heterosexual; mientras que el sexismo benevolente se constituye por el maternalismo, la diferenciación de género complementaria y la intimidad sexual (Rodríguez, Lameiras \& Carrera, 2009a).

Por lo tanto, según Rodríguez et al. (2009b), se podría decir que la teoría del sexismo ambivalente es un avance importante para tratar de conseguir igualdad entre hombres y mujeres, pues gracias a sus escalas se identifican las actitudes sexistas benevolentes y hostiles que representan daños y de esta manera puedan ser combatidas teniendo como objetivo una realidad equitativa entre los dos géneros.

Ahora bien, gran parte de la literatura disponible sobre sexismo ambivalente tiene dos características: 1) la evaluación del sexismo hacia las mujeres y, 2) el uso de poblaciones universitarias (León-Ramírez \& Ferrando, 2013; Zawisza, Luyt \& Zawadzka, 2012). Por ello es entendible que el número de estudios sobre sexismo ambivalente en edades más tempranas, como la adolescencia, presenten un número limitado.

En ese sentido podemos destacar una investigación con adolescentes gallegos que ha mostrado que los hombres son más sexistas hacia las mujeres y tienen mayores actitudes benevolentes hacia los miembros de su mismo sexo (Lameiras \& Rodríguez, 2003). En el mismo sentido puede mencionarse otro trabajo un poco más reciente con 
adolescentes españoles, donde se halló que los hombres son más sexistas hacia las mujeres y tienen mayores actitudes benevolentes hacia los hombres; por su parte las mujeres mostraron actitudes más hostiles hacia los hombres (Rodríguez, Lameiras, Carrera \& Faílde, 2010).

\section{Justificación}

Gran parte de las investigaciones realizadas, hasta el momento, están mayormente encaminadas a detectar el sexismo hacia las mujeres omitiendo la detección del sexismo hacia los hombres, quienes también forman parte del problema y solución a las desigualdades e iniquidades de género. Añadido a ello las actitudes y posturas sexistas de mujeres hacia hombres, especialmente en adolescentes son un tema del cual se tiene muy poca información.

Debido a que los pocos estudios realizados sobre este fenómeno en México evalúan sexismo hacia las mujeres, en este trabajo exploratorio nos proponemos identificar las actitudes sexistas hacia los hombres adolescentes esperando poder contribuir al aumento de los antecedentes disponibles en países hispanoparlantes sobre sexismo hacia los hombres.

Objetivo: Evaluar las actitudes hostiles y benevolentes hacia los hombres en adolescentes mexicanos.

\section{Método}

Estudio exploratorio, transversal y cuantitativo.

\section{Participantes}

Cuarenta y tres mujeres y treinta tres hombres, procedentes del Estado de Puebla (México), con edades comprendidas entre 12 y 19 años $(M=16.61, D T=1.20)$. La muestra fue no probabilística y por conveniencia.

\section{Instrumentos}

Se implementó la versión reducida de las escalas de Sexismo Ambivalente hacia Hombres, validada para población española por Rodríguez et al. (2009a), por lo cual se adaptaron algunos términos al español usado en México. La versión reducida de la escala está formada por 12 ítems, de los cuales 6 evalúan las actitudes hostiles hacia los hombres y los restantes, actitudes benevolentes. La puntuación de cada reactivo se obtiene mediante una escala Likert de 5 puntos $(0=$ Totalmente en desacuerdo, 1=Bastante en desacuerdo, 2=Algo en desacuerdo, 3=Algo de acuerdo, 4=Bastante de acuerdo 5=Totalmente de Acuerdo). A mayor puntuación, mayor nivel de sexismo. La fiabilidad de la escala Hostilidad hacia Hombres obtenida en este estudio fue de .81 para mujeres; y .67, para hombres; mientras que en la escala de Actitud Benevolente se obtuvo un coeficiente Alpha de .77 para mujeres; y .82, en la muestra masculina.

\section{Procedimiento}

Después de obtener el permiso de las autoridades académicas así como de la Comisión de ética de dos bachilleratos públicos del Estado de Puebla (México), las y los participantes contestaron el cuestionario vía on-line disponible desde Google docs. En la primera página se exponía la naturaleza y objetivos del estudio así como un ítem 
obligatorio que solicitaba la aceptación voluntaria y anónima para participar en el estudio. Los datos obtenidos se analizaron con el programa estadístico SPSS v. 19.

\section{Resultados}

Las medias obtenidas en las actitudes sexistas dan lugar a los resultados que a continuación se detallan.

Cuando se compararon las puntuaciones obtenidas por varones y mujeres en la subescala de sexismo benevolente se hallaron diferencias estadísticamente significativas $\left[t(70.9)=-3.18, p=.002\right.$, eta $\left.{ }^{2}=.11\right]$ entre lo manifestado por hombres $(M=17.04, D=6.48)$ y hombres $(M=12.74, D T=5.47)$.

Empero, no se hallaron diferencias significativas $[t(77.26)=.33, p=.74]$ en lo concerniente al sexismo hostil hacia los hombres, entre lo manifestado por las mujeres $(M=14.16, D T=5.99)$ y varones $(M=13.73, D T=5.67)$.



Figura 1. Puntuaciones medias de sexismo hacia los hombres

\section{Discusión}

Los resultados obtenidos en el presente trabajo están en concordancia con los resultados de investigaciones realizadas por Lameiras y Rodríguez (2003), quienes sugieren que a mayor nivel educativo menores niveles de sexismo se presentan en los adolescentes, mientras que Rodríguez et al. (2010) señalan que mayores edades se asocian con menores niveles de sexismo.

En el entendido que el sexismo es un fenómeno que permea tanto en hombres como mujeres se hace necesario seguir investigando las actitudes sexistas por medio del ASI y AMI, y cuál es el papel del sexismo benevolente y hostil en el mantenimiento de la desigualdad de género y la legitimación de la discriminación. En nuestra investigación los niveles de sexismo hostil son relativamente bajos, pero no así los niveles de sexismo benevolente donde destacan las puntuaciones obtenidos por hombres. Llaman la atención estos puntajes porque los niveles altos de sexismo benevolente hacia el hombre han sido asociados con la evaluación positiva y justificación de las relaciones tradicionales de género (Rollero \& Fedi, 2012) así como la aceptación de mitos de violación (Chapleau, Oswald \& Russell, 2007). 
Por lo anterior, abogamos por la necesidad de seguir investigando el sexismo hacia las mujeres, pero también hacia los hombres, en aras de lograr un trato teórico y científico igualitario que se traduzca en condiciones reales de equidad e igualdad entre ambos sexos, sobre todo para entender de manera más completa las dinámicas subyacentes a la discriminación hacia cada uno de los sexos.

Entre las limitaciones de este estudio se destaca que la muestra no fue seleccionada de forma probabilística, además de que su tamaño impide generalizar los resultados a otras poblaciones adolescentes en México. Por otra parte sería conveniente que dentro de las investigaciones venideras se amplíen y diversifiquen las características de la muestra, pues ciertamente el sexismo hacia el hombre y la mujer puede atravesar diferentes estratos socioeconómicos o etarios, además es preciso controlar la deseabilidad social de las respuestas.

\section{Referencias}

Chapleau, K.M., Oswald, D.L., \& Russell, B.L. (2007). How ambivalent sexism toward women and men support rape myth acceptance. Sex Roles, 57, 131-136. doi:10.1007/s11199-007-9196-2

Díaz-Aguado, M.J. (2003). Adolescencia, sexismo y violencia de género. Papeles del Psicólogo, 23(84), 35-44.

Glick, P., \& Fiske, S.T. (1996). The Ambivalent Sexism Inventory: Differentiating Hostile and Benevolent Sexism. Journal of Personality and Social Psychology, 70(3), 491-512. doi:10.1037/0022-3514.70.3.491

Glick, P., \& Fiske, S.T. (1999). The Ambivalence toward Men Inventory: Differentiating hostile and benevolent beliefs about men. Psychology of Women Quarterly, 23, 519-536. doi:10.1111/j.1471-6402.1999.tb00379.x

Lameiras, M. (2002). El sexismo y sus dos caras: De la hostilidad a la ambivalencia. Anuario de Sexología, 8, 91-102.

Lameiras, M., \& Rodríguez, Y. (2003). Evaluación del sexismo ambivalente en estudiantes gallegos/as. Acción psicológica, 2(2), 131-136.

León-Ramírez, B., \& Pere, F. (2013). Assessing sexism in a sample of Mexican students: A validity analysis based on the Ambivalent Sexism Inventory. Anuario de Psicología, 43(3), 335-347.

Rodríguez, Y., Lameiras, M., \& Carrera, M.V. (2009a). Validación de la versión reducida de las escalas ASI y AMI en una muestra de estudiantes españoles. Psicogente, 12(22), 284-295.

Rodríguez, Y., Lameiras, M., Carrera, M.V., \& Faílde, J.M. (2009b). Aproximación conceptual al sexismo ambivalente: Estado de la cuestión. SUMMA Psicológica UST, 6(2), 131-142.

Rodríguez, Y., Lameiras, M., Carrera, M.V., \& Faílde, J.M. (2010). Evaluación de las actitudes sexistas en estudiantes españoles/as de educación secundaria obligatoria. Psychologia. Avances de la disciplina, 4(1), 11-24. 
Rollero, C., \& Fedi, A. (2012). Ambivalent attitudes toward women and men. Psicología Política, 44, 69-86.

Zawisza, M., Luyt, R., \& Zawadzka, A.M. (2012). Ambivalence Toward Men: Comparing Sexism Among Polish, South African and British University Students. Sex Roles, 66, 453-467. doi:10.1007/s11199-011-0112-4.

Citar:

Arenas-Rojas, A.A., \& Rojas-Solis, J.L. (2015). Sexismo ambivalente hacia hombres: Un estudio exploratorio con adolescentes mexicanos. ReiDoCrea, 4, 54-59. http://digibug.ugr.es/handle/10481/33685

http://www.ugr.es/ reidocrea/ReiDoCrea-Vol.4-Art.8-Arenas.pdf 